\title{
Lodo de esgoto compostado e diferentes lâminas de irrigações no desenvolvimento de Acacia polyphylla
}

\author{
Nayara Gabriele Alcassa Alves Lanzetib ${ }^{\oplus}$, Ana Cláudia Chimini ${ }^{\mathrm{b}}$, Miguel Stancare Neto ${ }^{\mathrm{b}}{ }^{\circledR}$, Mar-

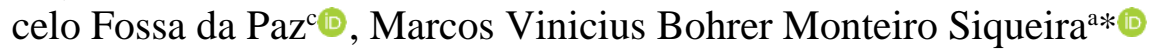 \\ a Universidade do Estado de Minas Gerais (Unidade Frutal), Brasil \\ b Universidade do Sagrado Coração, Brasil \\ c Universidade Federal da Grande Dourados, Brasil \\ *Autor correspondente (mvbsiqueira@gmail.com)
}

\section{N F O}

\section{Keyworks}

biosolids

native species

seedling production

nurseries

\begin{abstract}
A B S T R A C T
Composted sewage sludge and different irrigation layers on Acacia polyphylla development.

The selection of organic components to substrates in forest nurseries is increasingly common, with the aim of enhancing biological, chemical and physical data. The main components are the sewage process that goes through a stabilization process, becoming a rich component in industry that can be used in agriculture, and becomes a rich component in the composition of substrates. The objective of this work is to analyze the behavior of the species in different substrate compositions and irrigation depths. Five distinct formulations of composite substrate and commercial substrate (SC) and composted sludge and sewage (LEC) were used. The slide and irrigation evaluation was divided into three different irrigations, a total of 300 samples were analyzed. The parameters used were: plant height, stem diameter, leaf number, dry mass and root dry mass. Based on the results, the best alternatives for producing seedlings of wine in nurseries are observed, consisting of the following formulations: S1 (100\% LEC), S2 (25\% SC + 75\% LEC), and S3 (50\% SC + 50\% LEC). The combination of 100\% LEC resulted in the best development of seedlings, expressing a contribution with the increase in stem diameter, plant height, greater initial growth and root development, that is, in addition to the potential as fertilizer, it acts as a conditioner for physical assignments, chemical and biological necessary for seedlings. However, the different irrigation depths do not minimize the influences in any of the upper stages.
\end{abstract}

\section{R E S U M O}

É cada vez mais comum a adição de componentes orgânicos aos substratos em viveiros florestais, com o objetivo de potencializar seus atributos biológicos, químicos e físicos. Entre esses componentes destacase o lodo de esgoto que, após passar por processo de estabilização, torna-se um componente rico em matéria orgânica, podendo ser utilizado na agricultura, e em viveiros florestais. $\mathrm{O}$ trabalho teve como objetivo analisar o comportamento da espécie em diferentes composições de substratos e lâminas de irrigação. Utilizaram-se 5 formulações distintas de substrato compostas por substrato comercial (SC) e lodo e esgoto compostado (LEC). Avaliaram-se simultaneamente 3 lâminas de água, perfazendo um total de 300 mudas no estudo. Os parâmetros analisados foram: altura de planta, diâmetro do coleto, número de folhas, massa seca de parte aérea e raiz. Observou-se que os melhores tratamentos consistiram nas seguintes formulações: S1 (100\% LEC), S2 (25\% SC + 75\% LEC), e S3 (50\% SC + 50\% SC). Mostrou-se que, a partir do LEC puro, houve melhor desenvolvimento das mudas, expressando um aporte com o aumento no diâmetro do coleto, altura da planta, maior crescimento inicial e desenvolvimento radicular, ou seja, além do potencial como fertilizante, o LEC atua como condicionador de atribuições físicas, química e biológicas necessárias às mudas. As diferentes lâminas de irrigação não apresentaram influência significativa em nenhum dos parâmetros avaliados. 


\section{INTRODUÇÃO}

Acacia polyphylla DC (Fabaceae) é uma espécie arbórea conhecida pelos nomes comuns de monjoleiro e angico-branco. Sua distribuição geográfica vai desde a região Amazônica até o Paraná, sendo particularmente frequente nos Estados do Mato Grosso do Sul e São Paulo. Trata-se de uma árvore de porte médio, rápido crescimento e que pode atingir os $20 \mathrm{~m}$ de altura (Araújo-Neto et al., 2005). Sua floração acontece nos meses de dezembro a março, e os frutos expressam uma coloração em diferentes tons de marrom. Possui folhas compostas e bipinadas, apresentando de 24 a 34 pares de folíolos (Araújo-Neto et al., 2002). Apresenta grande potencial para recuperação de áreas degradadas e áreas de preservação permanente, além de ser usada em projetos paisagísticos.

Por ser uma leguminosa do gênero Acacia apresenta grande potencial de fixação de nitrogênio (Chaer et al., 2011). É classificada como uma espécie pioneira de rápido crescimento, com boa capacidade de competição com plantas de fotossíntese $\mathrm{C} 4$ e alta taxa de sobrevivência das mudas, com apenas $17 \%$ de mortalidade nos primeiros dois anos (Campoe et al., 2014).

Dentro desse contexto e, tendo em vista o grande potencial da espécie, torna-se essencial que o manejo e o processo de produção de mudas sejam adequados. Nos viveiros, a qualidade da muda depende dos materiais e do manejo utilizado, principalmente do substrato, já que esse é o responsável pela sustentação da planta e sua fonte de água e nutrientes.

Os estudos mostram que tem se tornado comum o uso de diferentes componentes orgânicos em substratos de viveiros florestais, com o objetivo de potencializar seus atributos biológicos, químicos e físicos (Delarmelina et al., 2014; Trigueiro e Guerrini, 2014). Trazzi et al. (2012) reportaram que o substrato deve conter caraterísticas fundamentais, destacando-se a estrutura, consistência, boa porosidade, capacidade de retenção de água, estar livre de substâncias tóxicas, plantas invasoras, insetos e doenças.

Por outro lado, a expansão das cidades acarreta inúmeros problemas ambientais, dentre os quais a geração de um grande volume de lodo de esgoto, que após seu tratamento, estabilização e compostagem, dão origem ao lodo de esgoto compostado (LEC) (Cabreira et al., 2017). O lodo de esgoto resulta do esgoto recolhido das residências, indústrias e das Estações de Tratamento de Esgoto.

Quanto maior o investimento em saneamento

básico, maior será a coleta e tratamento de esgoto, resultando em maiores quantidades de $\operatorname{LEC}(\mathrm{Ca}$ margo et al., 2013).
Para Scheer et al. (2010), um dos resíduos promissores para uso em viveiros é exatamente o LEC, visto apresentar altos teores de nutrientes, que além de possibilitar uma diminuição de alguns problemas ambientais causados pelo lodo como aumento de doenças por veiculação hídrica (Neves et al., 2019), modificação da atividade microbiana do solo (Moreira et al., 2019) e deposição de metais pesados (Chu e He, 2020), proporciona uma economia na adubação suplementar de viveiros, ou seja, seu custo torna-se inferior em comparação aos fertilizantes minerais sintéticos e substratos comerciais amplamente utilizados (Vieira e Weber, 2016).

Neste contexto, Scheer et al. (2012) e Delarmelina et al. (2014) pontuam que, visando uma produção de qualidade por parte dos viveiros, o LEC pode ser utilizado como condicionador de atribuições físicas, química e biológicas necessárias às mudas. Cabreira et al. (2017) reforçam que a destinação correta do LEC deve ser uma alternativa altamente promissora, viável e acima de tudo sustentável.

Delarmelina et al. (2014), ressaltam que uma grande diversidade de compostos pode ser incorporados ao substrato (dentre eles o LEC), porém, é necessário um estudo detalhado sobre a eficácia destes. Para que ocorra a estimulação do crescimento inicial das mudas, bem como nas fases seguintes, esses compostos devem trazer todas as condições necessárias ao bom desenvolvimento, sendo essa a fase mais importante nos viveiros (Trazzi et al., 2012).

Além dos substratos, outro fator de alta importância é a irrigação para o bom desenvolvimento das mudas. As irrigações em viveiros são realizadas manualmente, com regadores ou mangueiras, por aspersão, e por micro aspersão. Este último tem sido mais indicado em função da economia, da mão-de-obra e do maior controle sobre a distribuição da água.

$\mathrm{Na}$ irrigação dos canteiros de semeadura e das mudas em estágio inicial de desenvolvimento, as regas devem ser mais frequentes do que para as mudas já desenvolvidas. $\mathrm{O}$ excesso de rega costuma ser mais prejudicial do que a falta por dificultar a circulação de ar no solo, impedindo o crescimento das raízes, lixiviação dos nutrientes e o aparecimento de doenças (Testezlaf, 2017).

$O$ presente trabalho teve como objetivo analisar o comportamento da espécie A. Polyphylla em diferentes formulações de substratos e lâminas de irrigação.

\section{MATERIAL E MÉTODOS}

\section{Local}

O presente trabalho foi desenvolvido no Viveiro 
da Associação de Recuperação Florestal e Ecológica (ACIFLORA), localizado na cidade de Bauru, SP. Segundo a classificação de Koppen (Alvares et al., 2013), o clima da região é tropical de savana, tipo Aw, com uma média de precipitação anual de $1.331 \mathrm{~mm}$ e de temperatura com máxima de $32{ }^{\circ} \mathrm{C}$ e mínima de $20^{\circ} \mathrm{C}$.

\section{Montagem do Experimento}

A quebra de dormência das sementes foi realizada através do processo de choque térmico a 100 ${ }^{\circ} \mathrm{C}$ por um período de dois a três minutos e seguidamente secas em papel toalha para a retirada da umidade excedente e ambientação. Posteriormente foram semeadas em canteiros com areia e cobertos por vermiculita. Ao adquirir o tamanho de aproximadamente $1,5 \mathrm{~cm}$, procedeu-se a repicagem para tubetes individuais de $120 \mathrm{~cm}^{3}$.

Manualmente preparados, os substratos foram inseridos nos tubetes e depositados nas grades de apoio das bancadas permanecendo em casa de vegetação por quatorze dias. O experimento, já estabelecido em casa de vegetação e mantido em ambiente aberto foi organizado em três lâminas com quatro parcelas cada - repetições com dez tubetes para cada tratamento. Sendo assim, cada lâmina de água apresentou 200 indivíduos.

\section{Substrato}

No delineamento experimental, os substratos utilizados foram o Carolina Soil Florestal ${ }^{\circledR}$ identificados como SC e constituído por turfa de Sphagno, vermiculita expandida, casca de arroz carbonizada, traços de NPK, calcário dolomítico, e gesso agrícola; e o composto orgânico Terafertil, identificado como substrato de LEC, fornecido pela empresa Tera Ambiental do município de Jundiaí-SP. A partir de ambos os substratos, resultaram cinco substratos diferentes: $\mathrm{S} 1=100 \%$ LEC; $\mathrm{S} 2=75 \%$ LEC $+25 \% \mathrm{SC} ; \mathrm{S} 3=50 \% \mathrm{LEC}+50 \% \mathrm{SC} ; \mathrm{S} 4=25 \%$ $\mathrm{LEC}+75 \% \mathrm{SC} ;$ e S5=100\% SC.

\section{Irrigação}

Utilizou-se lâminas (L) distintas: L1 = irrigação de 6 minutos; a L2= irrigação de 9 minutos; e L3 = irrigação de 12 minutos; todas quatro vezes ao dia. Cada irrigação correspondeu às seguintes quantidades de água: $36 \mathrm{~mL} / \mathrm{dia}, 72 \mathrm{~mL} / \mathrm{dia}$, e $108 \mathrm{~mL} / \mathrm{dia}$, respectivamente. Foi empregado um sistema de irrigação por micro aspersão com aspersores fixo de giro completo $\left(360^{\circ}\right)$.

\section{Avaliações biométricas}

$\mathrm{Na}$ avaliação das mudas, estas foram mesurados durante 10 meses (uma medição por mês), os parâmetros avaliados foram: altura da parte aérea, diâmetro do coleto e número de folhas das mudas. As medições foram realizadas com o auxílio de paquímetro digital, com valores expressos em milímetro.

Após o término das medições, para a avaliação da massa seca de parte aérea e a massa seca de raiz, foram separadas as partes aéreas das raízes as quais foram colocadas em sacos de papel separadamente. Em uma primeira fase, foram realizadas as pesagens do material fresco, e após os sacos permanecerem em estufa em circulação forçada à temperatura de $62{ }^{\circ} \mathrm{C}$ pelo período de $48 \mathrm{~h}$, realizaram-se a pesagem da matéria seca.

A combinação das variáveis 'substrato' (S1 a S5) e 'lâmina de água' (L1 a L3) gerou 15 tratamentos, divididos em 4 parcelas, com 10 repetições dispostas em casa de vegetação de forma a reduzir as demais variáveis ambientais.

\section{Análise estatística}

Os dados foram analisados por análise multivariada e comparados pelo teste de Tukey através do programa WinStat.

A velocidade de crescimento foi calculada pelo coeficiente angular obtido a partir da regressão linear de cada tratamento (combinação de substrato com lâminas de água).

\section{RESULTADOS E DISCUSSÃO}

Observa-se na Tabela 1 que aos 300 dias o S3 apresentou resultados superiores em relação aos demais, inclusive S5 (100\% SC - considerando aqui o tratamento controle), seguido dos substratos S2 e S4, porém sem significância estatística.

Já quando avaliada a curva de crescimento no decorrer dos 300 dias, o substrato que apresentou maior taxa de crescimento (determinada pelo coeficiente angular) foi a combinação S4 com taxa de crescimento de $1,21 \mathrm{~mm} / \mathrm{dia}$, seguido de $\mathrm{S} 3 \mathrm{com}$ $1,17 \mathrm{~mm} /$ dia, em S2 com taxa de crescimento de $1,16 \mathrm{~mm} /$ dia, e $\mathrm{S} 1$ e $\mathrm{S} 5$, ambos com $1,11 \mathrm{~mm} / \mathrm{dia}$. Assumiu-se o grau de confiabilidade acima de $99,61 \%$ para todas as curvas.

De acordo com Gomes e Paiva (2013), a altura da parte aérea é um ótimo parâmetro biométrico para uma perspectiva do crescimento inicial de mudas em campo, tratando-se de um atributo de fácil medição, cujo método de obtenção é do tipo não destrutivo, podendo também ser utilizado para estimar o padrão de qualidade de mudas de espécies florestais nos viveiros. No entanto, para Rudek et al. (2013), a altura das mudas não é tecnicamente uma boa variável para tal avaliação de qualidade, considerando que as mudas podem passar por diversas variações ambientais, a exemplo dos níveis nutricionais, o qual oscila em conjunto com a fertilização fornecida, podendo causar o aumento nos teores de nitrogênio pela adubação ocasionando o 
estiolamento da muda. Dessa forma, o presente trabalho admite a altura das mudas como uma variável confiável, visto que todo o processo de criação das mudas, desde o preparo dos substratos até ao último dia de permanência no viveiro foram monitorados, ou seja, não foram expostas a variações ambientais citadas.

Tabela 1 - Altura das mudas de A. polyphylla após a repicagem até a medição final por tratamento.

Altura das mudas (cm)

\begin{tabular}{ccccccccccccc}
\hline $\begin{array}{c}\text { Subst } \\
\text { ratos }\end{array}$ & $\begin{array}{c}30 \\
\text { dias }\end{array}$ & $\begin{array}{c}60 \\
\text { dias }\end{array}$ & $\begin{array}{c}90 \\
\text { dias }\end{array}$ & $\begin{array}{c}120 \\
\text { dias }\end{array}$ & $\begin{array}{c}150 \\
\text { dias }\end{array}$ & $\begin{array}{c}180 \\
\text { dias }\end{array}$ & $\begin{array}{c}210 \\
\text { dias }\end{array}$ & $\begin{array}{c}240 \\
\text { dias }\end{array}$ & $\begin{array}{c}270 \\
\text { dias }\end{array}$ & $\begin{array}{c}300 \\
\text { dias }\end{array}$ & $\begin{array}{c}\text { Cres. } \\
(\mathrm{mm} \\
\left.\text { dia }^{-1}\right)\end{array}$ & $\mathrm{R}^{2}$ \\
\hline \multirow{2}{*}{$\mathrm{S} 1$} & $0,60 \pm$ & $1,09 \pm$ & $1,52 \pm$ & $1,86 \pm$ & $2,16 \pm$ & $2,46 \pm$ & $2,78 \pm$ & $3,11 \pm$ & $3,46 \pm$ & $3,80 \pm$ & 1,11 & 0,99 \\
& 0,21 & 0,20 & 0,17 & 0,17 & 0,15 & 0,15 & 0,15 & 0,16 & 0,18 & 0,17 & 71 \\
S2 & $0,60 \pm$ & $1,09 \pm$ & $1,51 \pm$ & $1,87 \pm$ & $2,21 \pm$ & $2,54 \pm$ & $2,88 \pm$ & $3,22 \pm$ & $3,57 \pm$ & $3,91 \pm$ & 1,16 & 0,99 \\
& 0,20 & 0,20 & 0,15 & 0,14 & 0,15 & 0,14 & 0,13 & 0,12 & 0,13 & 0,14 & 72 \\
S3 & $0,62 \pm$ & $1,11 \pm$ & $1,53 \pm$ & $1,91 \pm$ & $2,27 \pm$ & $2,62 \pm$ & $2,97 \pm$ & $3,31 \pm$ & $3,64 \pm$ & $3,97 \pm$ & 1,17 & 0,99 \\
& 0,14 & 0,14 & 0,13 & 0,12 & 0,13 & 0,12 & 0,11 & 0,11 & 0,11 & 0,12 & 56 \\
S4 & $0,5 \pm$ & $1,09 \pm$ & $1,54 \pm$ & $1,92 \pm$ & $2,27 \pm$ & $2,62 \pm$ & $2,96 \pm$ & $3,28 \pm$ & $3,60 \pm$ & $3,91 \pm$ & 1,21 & 0,99 \\
& 0,21 & 0,16 & 0,15 & 0,13 & 0,13 & 0,12 & 0,12 & 0,12 & 0,12 & 0,13 & 56 \\
S5 & $0,52 \pm$ & $1,01 \pm$ & $1,45 \pm$ & $1,83 \pm$ & $2,17 \pm$ & $2,50 \pm$ & $2,82 \pm$ & $3,12 \pm$ & $3,41 \pm$ & $3,68 \pm$ & 1,11 & 0,99 \\
& 0,19 & 0,16 & 0,15 & 0,16 & 0,16 & 0,14 & 0,14 & 0,14 & 0,14 & 0,16 & 22 \\
\hline
\end{tabular}

$\mathrm{S} 1=100 \%$ LEC, S2 $=75 \%$ LEC $+25 \%$ SC, S3 $=50 \%$ LEC $+50 \%$ SC, S4= 25\% LEC $+75 \%$ SC e S5 $=100 \%$ SC.

. Substituir o trecho cortado por:

Para a variável altura a melhor combinação foi a S4 seguida de S3 e S2 demonstrando melhores resultados para substratos com menos LEC, porém com melhor desempenho que o padrão (SC). O melhor resultado para $\mathrm{S} 4$ se deve ao equilíbrio entre a macroporosidade, atributo conferido pelo SC e o teor de nutrientes advindo do LEC.

Provavelmente essa condição se deve ao equilíbrio de 2 fatores: (i) a macroporosidade e (ii) o teor de nutrientes. Assim, os extremos, SC e LEC puro, apresentaram desvantagens em relação às suas misturas já que o complexo traz equilíbrio entre aeração, promovida pelo substrato SC e incremento de nutrientes pelo LEC. Resultados semelhantes foram observados por Lobo et al. (2018), com a espécie quaresmeira (Tibouchina granulosa). Os autores utilizaram, composições idênticas às do pre- sente estudo (as mesmas cinco misturas de substratos de LEC e SC) e verificaram que é viável a utilização da combinação de LEC e SC para favorecer o desenvolvimento da espécie, porém para quaresmeira, a mistura de melhor desempenho foi equivalente ao substrato S2, seguida do S3 e S4.

Quanto às lâminas de água avaliadas isoladamente, não ocorreram variações estatísticas significativas entre as três lâminas (Tabela 2). As médias apresentaram resultados semelhantes entre si, podendo-se considerar que a quantidade de água utilizada em cada lâmina, bem como os diferentes tempos de irrigação, não afetou a efetividade do SC e do LEC nas mudas de A. polyphylla. Apesar disso, quando avaliada a curva de crescimento, há uma leve tendência positiva na irrigação L2 com velocidade de crescimento de $1,15 \mathrm{~mm} /$ dia, seguido da $\mathrm{L} 1$ com 1,18 mm/dia e por L3 com 1,15 mm/dia.

Tabela 2 - Altura das mudas de A. polyphylla após a repicagem até a medição final por lâmina de irrigação.

\begin{tabular}{|c|c|c|c|c|c|c|c|c|c|c|c|c|}
\hline \multicolumn{13}{|c|}{ Altura das mudas $(\mathrm{cm})$} \\
\hline $\begin{array}{c}\text { Lâmi- } \\
\text { nas }\end{array}$ & $\begin{array}{c}30 \\
\text { dias }\end{array}$ & $\begin{array}{c}60 \\
\text { dias }\end{array}$ & $\begin{array}{c}90 \\
\text { dias }\end{array}$ & $\begin{array}{l}120 \\
\text { dias }\end{array}$ & $\begin{array}{l}150 \\
\text { dias }\end{array}$ & $\begin{array}{l}180 \\
\text { dias }\end{array}$ & $\begin{array}{l}210 \\
\text { dias }\end{array}$ & $\begin{array}{l}240 \\
\text { dias }\end{array}$ & $\begin{array}{l}270 \\
\text { dias }\end{array}$ & $\begin{array}{l}300 \\
\text { dias }\end{array}$ & $\begin{array}{l}\text { Cres. } \\
(\mathrm{mm} \\
\left.\mathrm{dia}^{-1}\right)\end{array}$ & $\mathrm{R}^{2}$ \\
\hline $\mathrm{L} 1$ & $\begin{array}{c}0,57 \pm \\
0,03\end{array}$ & $\begin{array}{c}1,05 \pm \\
0,03\end{array}$ & $\begin{array}{c}1,45 \pm \\
0,03\end{array}$ & $\begin{array}{c}1,80 \pm \\
0,02\end{array}$ & $\begin{array}{c}2,17 \pm \\
0,02\end{array}$ & $\begin{array}{c}2,49 \pm \\
0,02\end{array}$ & $\begin{array}{c}2,80 \pm \\
0,02\end{array}$ & $\begin{array}{c}3,12 \pm \\
0,02\end{array}$ & $\begin{array}{c}3,43 \pm \\
0,02\end{array}$ & $\begin{array}{c}3,75 \pm \\
0,02\end{array}$ & 1,15 & 0,9959 \\
\hline $\mathrm{L} 2$ & $0,58 \pm 0,03$ & $\begin{array}{c}1,08 \pm \\
0,02\end{array}$ & $\begin{array}{c}1,51 \pm \\
0,01\end{array}$ & $\begin{array}{c}1,88 \pm \\
0,01\end{array}$ & $\begin{array}{c}2,21 \pm \\
0,02\end{array}$ & $\begin{array}{c}2,55 \pm \\
0,02\end{array}$ & $\begin{array}{c}2,88 \pm \\
0,01\end{array}$ & $\begin{array}{c}3,21 \pm \\
0,02\end{array}$ & $\begin{array}{c}3,53 \pm \\
0,02\end{array}$ & $\begin{array}{c}3,85 \pm \\
0,03\end{array}$ & 1,18 & 0,9955 \\
\hline L3 & $0,54 \pm 0,03$ & $\begin{array}{c}1,00 \pm \\
0,04\end{array}$ & $\begin{array}{c}1,41 \pm \\
0,03\end{array}$ & $\begin{array}{c}1,76 \pm \\
0,03\end{array}$ & $\begin{array}{c}2,09 \pm \\
0,03\end{array}$ & $\begin{array}{c}2,41 \pm \\
0,02\end{array}$ & $\begin{array}{c}2,74 \pm \\
0,02\end{array}$ & $\begin{array}{c}3,07 \pm \\
0,03\end{array}$ & $\begin{array}{c}3,39 \pm \\
0,03\end{array}$ & $\begin{array}{c}3,71 \pm \\
0,04\end{array}$ & 1,15 & 0,9971 \\
\hline
\end{tabular}

S1 $=100 \%$ LEC, S2 $=75 \%$ LEC + 25\% SC, S3= 50\% LEC + 50\% SC, S4= 25\% LEC + 75\% SC e S5= 100\% SC. L1= irrigação de 6 minutos, L2= irrigação 9 minutos e L3= irrigação e 12 minutos.

Quando avaliados conjuntamente, os resultados 
L2/S3, L2/S2 e L2/S4 (Figura 01). Apesar de também não apresentarem significância estatística entre as primeiras combinações, os melhores resultados se mantêm entre as L2 e L3 com três das quatro maiores médias para a L2, irrigação intermediária, e o S4 com 2 das 4 maiores médias. Assim, confirma-se que a L2 e o S4 são mais frequentes entre os melhores resultados, indicando essas duas variáveis como preferenciais. Nas 4 melhores médias não aparecem os substratos S1 nem S5 indicando, novamente, que os extremos são desfavoráveis ao desenvolvimento das mudas.

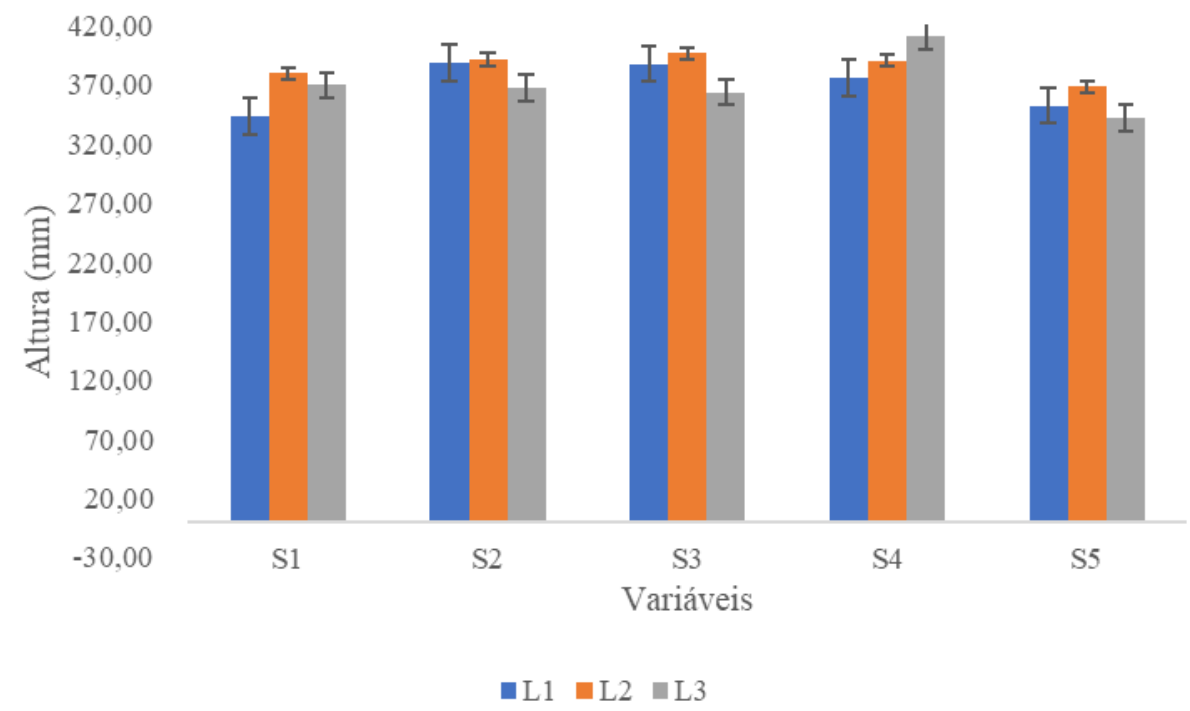

Figura 1 - Altura das mudas de A. polyphylla com 300 dias. Combinação entre substrato e lâmina de irrigação. $\mathrm{S} 1=100 \%$ LEC, S $2=75 \%$ LEC + $25 \%$ SC, S $3=50 \%$ LEC + 50\% SC, S $4=25 \%$ LEC + $75 \%$ SC e S $5=100 \%$ SC. L1= irrigação de 6 minutos, L2= irrigação 9 minutos e L3= irrigação e 12 minutos.

A linearidade no crescimento das mudas, quando se avalia a variável lâmina de água isoladamente, indica que até 300 dias não há necessidade de alteração na irrigação.
A avaliação do diâmetro do coleto apresentou resultados melhores nos substratos, S2 e S1. S5 apresentou resultados inferiores durante todo o experimento (Tabela 3).

Tabela 3 - Avaliação do diâmetro de coleto em mudas de A. polyphylla.

\begin{tabular}{ccccccccccc}
\hline \multicolumn{10}{c}{ Diâmetro do coleto das mudas (mm) } \\
\hline \multirow{2}{*}{ Substratos } & $\begin{array}{c}30 \\
\text { dias }\end{array}$ & $\begin{array}{c}60 \\
\text { dias }\end{array}$ & $\begin{array}{c}90 \\
\text { dias }\end{array}$ & $\begin{array}{c}120 \\
\text { dias }\end{array}$ & $\begin{array}{c}150 \\
\text { dias }\end{array}$ & $\begin{array}{c}180 \\
\text { dias }\end{array}$ & $\begin{array}{c}210 \\
\text { dias }\end{array}$ & $\begin{array}{c}240 \\
\text { dias }\end{array}$ & $\begin{array}{c}270 \\
\text { dias }\end{array}$ & $\begin{array}{c}300 \\
\text { dias }\end{array}$ \\
\hline S1 & 1,00 & $1,58 \mathrm{~b}$ & $1,82 \mathrm{a}$ & $1,85 \mathrm{a}$ & $1,89 \mathrm{a}$ & $1,91 \mathrm{a}$ & $1,98 \mathrm{ab}$ & $2,02 \mathrm{ab}$ & $2,07 \mathrm{ab}$ & 2,11 \\
$\mathrm{~S} 2$ & 1,00 & $1,70 \mathrm{a}$ & $1,85 \mathrm{a}$ & $1,91 \mathrm{a}$ & $1,97 \mathrm{a}$ & $2,03 \mathrm{a}$ & $2,06 \mathrm{a}$ & $2,10 \mathrm{a}$ & $2.12 \mathrm{a}$ & 2,17 \\
$\mathrm{~S} 3$ & 1,00 & $1,66 \mathrm{ab}$ & $1,80 \mathrm{a}$ & $1,85 \mathrm{a}$ & $1,88 \mathrm{a}$ & $1,93 \mathrm{a}$ & $1,94 \mathrm{ab}$ & $1,97 \mathrm{ab}$ & $2,01 \mathrm{ab}$ & 2,03 \\
$\mathrm{~S} 4$ & 1,00 & $1,58 \mathrm{~b}$ & $1,81 \mathrm{a}$ & $1,85 \mathrm{a}$ & $1,90 \mathrm{a}$ & $1,92 \mathrm{a}$ & $1,98 \mathrm{ab}$ & $2,00 \mathrm{ab}$ & $2,04 \mathrm{ab}$ & 2,07 \\
$\mathrm{~S} 5$ & 1,00 & $1,37 \mathrm{c}$ & $1,61 \mathrm{~b}$ & $1,67 \mathrm{~b}$ & $1,72 \mathrm{~b}$ & $1,73 \mathrm{~b}$ & $1,80 \mathrm{~b}$ & $1,84 \mathrm{~b}$ & $1,88 \mathrm{~b}$ & 1,92 \\
\hline F \% & - & $26,37^{* *}$ & $9,11^{* *}$ & $5,96^{* *}$ & $5,01^{* *}$ & $6,97 * *$ & $4,20^{* *}$ & $3,44^{*}$ & $2,43 \mathrm{~ns}$ & $2,29 \mathrm{~ns}$ \\
DMS 5\% & - & 0,10 & 0,13 & 0,15 & 0,17 & 0,17 & 0,19 & 0,22 & 0,24 & 0,25 \\
CV \% & - & 5,42 & 6,15 & 7,21 & 7,64 & 7,57 & 8,23 & 9,26 & 9,99 & 10,52 \\
\hline
\end{tabular}

S1 $=100 \%$ LEC, S2 $=75 \%$ LEC e $25 \%$ SC, S3= 50\% LEC e 50\% SC, S4= 25\% LEC e 75\% SC e S5= 100\% SC. DMS diferença mínima significativa, CV coeficiente de variação e ns não significativo. * significativo ao nível de $5 \%$ de probabilidade $(01=<\mathrm{p}<.05)$; ** significativo ao nível de $1 \%$ de probabilidade $(\mathrm{p}<.01)$.

Gomes e Paiva (2013) relataram que o diâmetro 
do coleto é uma das características mais importantes em relação ao desenvolvimento de mudas de espécies arbóreas após o plantio em campo, sendo que, quanto mais alto for o valor do diâmetro de coleto de uma muda, melhor será a distribuição de fotoassimilados na sua parte aérea.

De acordo com Sturion e Antunes (2000) e Lobo et al. (2018), um dos parâmetros mais utilizados, na qualidade de mudas, para fins de reflorestamento, é a altura e o diâmetro do coleto, visto que asseguram a resistência e fixação no solo. No entanto, para a planta se manter ereta é preciso que apresente em sua estrutura alto diâmetro do coleto, pois caso contrário, resultará no tombamento e consequentemente na deformação ou morte da planta. Rudek et al. (2013), ressaltam ainda que, mesmo por sua facilidade de mensuração, deve-se levar esta variável como sendo uma das mais importante, entre outras avaliadas neste tipo de pesquisa.

Delarmelina et al. (2013) testando LEC em sesbânia-amarela (Sesbania virgata), demonstraram que, para melhores resultados em altura e diâmetro do coleto, é recomendado a utilização de compostos de dois diferentes substratos ao invés de compostos de tratamentos puros, visto que os maiores valores para diâmetro do coleto e melhor crescimento em altura foram obtidos por tratamentos que continham LEC e composto orgânico em sua composição. No entanto, os dados obtidos neste estudo divergem desta ideia, pois de acordo com os resultados na Tabela 03, tanto os compostos de dois substratos, quanto os compostos de tratamentos puros com LEC, influenciam positivamente na altura e diâmetro do coleto.

De acordo com a Tabela 04, nota-se que o diâmetro de coleto das mudas de A. polyphylla em relação às lâminas de irrigação, não apresentou diferença significativa após os 300 dias de condução do experimento.

Tabela 4 - Avaliação do diâmetro de coleto das mudas de A. polyphylla em relação às lâminas de irrigação

\begin{tabular}{|c|c|c|c|c|c|c|c|c|c|c|}
\hline \multicolumn{11}{|c|}{ Diâmetro do coleto (mm) } \\
\hline Lâminas & $\begin{array}{c}30 \\
\text { dias }\end{array}$ & $\begin{array}{c}60 \\
\text { dias }\end{array}$ & $\begin{array}{c}90 \\
\text { dias }\end{array}$ & $\begin{array}{l}120 \\
\text { dias }\end{array}$ & $\begin{array}{l}150 \\
\text { Dias }\end{array}$ & $\begin{array}{l}180 \\
\text { dias }\end{array}$ & $\begin{array}{l}210 \\
\text { dias }\end{array}$ & $\begin{array}{l}240 \\
\text { dias }\end{array}$ & $\begin{array}{l}270 \\
\text { dias }\end{array}$ & $\begin{array}{l}300 \\
\text { dias }\end{array}$ \\
\hline L1 & 1,00 & $1,49 b$ & 1,77 & $1,79 b$ & $1,84 \mathrm{~b}$ & 1,88 & $1,93 \mathrm{ab}$ & $1,96 a b$ & 2,00 & 2,03 \\
\hline L2 & 1,00 & $1,55 \mathrm{ab}$ & 1,76 & $1,80 \mathrm{~b}$ & $1,84 b$ & 1,87 & $1,90 \mathrm{~b}$ & $1,94 b$ & 1,98 & 2,00 \\
\hline L3 & 1,00 & $1,69 \mathrm{a}$ & 1,81 & $1,89 \mathrm{a}$ & $1,94 \mathrm{a}$ & 1,95 & $2,03 \mathrm{a}$ & $2,07 \mathrm{a}$ & 2,1 & 2,14 \\
\hline $\mathrm{F} \%$ & - & $6,85^{*}$ & $1,78 \mathrm{~ns}$ & $7,00 *$ & $9,33^{*}$ & $2,02 \mathrm{~ns}$ & $6,43^{*}$ & $6,23 *$ & $3,45 \mathrm{~ns}$ & $4,42 \mathrm{~ns}$ \\
\hline DMS 5\% & - & 0,17 & 0,09 & 0,09 & 0,08 & 0,14 & 0,11 & 0,12 & 0,15 & 0,15 \\
\hline CV\% & - & 11,10 & 5,29 & 5,14 & 4,64 & 7,44 & 6,08 & 6,38 & 7,77 & 7,33 \\
\hline
\end{tabular}

L1= irrigação de 6 minutos, L2= irrigação de 9 minutos e L3= irrigação de 12 minutos.

DMS diferença mínima significativa, CV coeficiente de variação e não significativo.

* significativo ao nível de $5 \%$ de probabilidade $(01=<p<05)$.

Santana et al. (2019) analisaram a resposta do dedaleiro (Lafoensia pacari) a tratamentos com LEC e diferentes lâminas de água. Os resultados mostraram que o substrato com LEC puro apresentou desenvolvimento superior em relação aos demais tratamentos mediante a lâmina de $10 \mathrm{~L} \mathrm{dia}^{-1}$. Diferentemente dos resultados da Tabela 04, onde a lâmina com $108 \mathrm{~mL} /$ dia se destaca das lâminas de $36 \mathrm{~mL} / \mathrm{dia}$ e $72 \mathrm{~mL} / \mathrm{dia}$, apresentando diminuta disparidade.

$\mathrm{Na}$ avaliação foliar das mudas de A. polyphylla, os tratamentos demonstraram grandes diferenças estatísticas entre si ao longo das medições (Tabela 5). Observa-se que aos 30 dias S4 apresentou um maior número para este parâmetro comparando-se com os demais tratamentos; em contrapartida, aos 60 dias, S1 demonstrou grande evolução de crescimento foliar, apresentando os maiores valores neste parâmetro até 210 dias. A partir de 240 dias, os tratamentos S1, S2, S3, e S4 manifestaram valores significativos em relação à $\mathrm{S} 5$, o qual apresentou valores menores durante todo o experimento.

No presente experimento não ocorreram variações estatísticas no número de folhas em relação às diferentes lâminas e irrigação utilizadas, ou seja, a irrigação não influenciou significativamente no desenvolvimento foliar das mudas de A. polyphylla, mesmo as quantidades e o tempo sendo distintos entre si. 
$\underline{\text { Tabela } 5 \text { - Número de folhas das mudas de A. polyphylla após a repicagem até a medição final por tratamento }}$

Número de folhas

\begin{tabular}{|c|c|c|c|c|c|c|c|c|c|c|}
\hline Substratos & $\begin{array}{c}30 \\
\text { dias }\end{array}$ & $\begin{array}{c}60 \\
\text { Dias } \\
\end{array}$ & $\begin{array}{c}90 \\
\text { dias }\end{array}$ & $\begin{array}{l}120 \\
\text { dias }\end{array}$ & $\begin{array}{l}150 \\
\text { dias }\end{array}$ & $\begin{array}{l}180 \\
\text { dias }\end{array}$ & $\begin{array}{l}210 \\
\text { dias }\end{array}$ & $\begin{array}{l}240 \\
\text { dias }\end{array}$ & $\begin{array}{l}270 \\
\text { dias }\end{array}$ & $\begin{array}{l}300 \\
\text { dias }\end{array}$ \\
\hline S1 & $6,66 \mathrm{ab}$ & $9,70 \mathrm{a}$ & $11,87 \mathrm{a}$ & $12,56 \mathrm{a}$ & $11,80 \mathrm{a}$ & $12,29 a$ & $12,56 a$ & $12,8 \mathrm{a}$ & $12,54 \mathrm{a}$ & $12,45 \mathrm{a}$ \\
\hline S2 & $6,03 b$ & $8,45 \mathrm{c}$ & $10,37 b$ & $10,92 b$ & $10,37 \mathrm{bc}$ & $11,10 \mathrm{~b}$ & $12,01 \mathrm{ab}$ & $12,82 \mathrm{a}$ & $13,04 \mathrm{a}$ & $13,28 \mathrm{a}$ \\
\hline S3 & $6,63 a b$ & $8,91 b c$ & $10,61 b$ & $11,01 \mathrm{~b}$ & $10,82 \mathrm{ab}$ & $11,39 a b$ & $11,87 \mathrm{ab}$ & $11,71 \mathrm{a}$ & $11,62 \mathrm{a}$ & $11,62 \mathrm{a}$ \\
\hline S4 & $6,80 \mathrm{a}$ & $9,68 \mathrm{ab}$ & $11,04 a b$ & $10,84 b$ & $9,61 \mathrm{c}$ & $10,41 b$ & $11,12 b$ & $11,45 \mathrm{a}$ & $11,63 a$ & $11,52 \mathrm{a}$ \\
\hline S5 & $6,70 \mathrm{ab}$ & $8,16 \mathrm{c}$ & $9,96 \mathrm{~b}$ & $9,30 \mathrm{c}$ & $7,54 d$ & $8,38 \mathrm{c}$ & $9,05 \mathrm{c}$ & $9,42 \mathrm{~b}$ & $9,09 b$ & $8,69 \mathrm{~b}$ \\
\hline F \% & $2,64 *$ & $12,83^{* *}$ & $7,01 * *$ & $14,04 * *$ & $29,27 * *$ & $25,8 * *$ & $16,73^{* *}$ & $14,39 * *$ & $12,3 * *$ & $11,95 * *$ \\
\hline DMS 5\% & 0,75 & 0,79 & 1,12 & 1,24 & 1,20 & 1,17 & 1,36 & 1,49 & 1,76 & 2,03 \\
\hline CV \% & 9,79 & 7,51 & 8,87 & 9,76 & 10,22 & 9,34 & 10,25 & 10,91 & 12,97 & 15,06 \\
\hline
\end{tabular}

S1 $=100 \%$ LEC, S2 $=75 \%$ LEC + 25\% SC, S3= 50\% LEC + 50\% SC, S4= 25\% LEC + 75\% SC e S5= 100\% SC. DMS diferença mínima significativa, CV coeficiente de variação e não significativo. * significativo ao nível de $5 \%$ de probabilidade $(01=<\mathrm{p}<05)$; ** significativo ao nível de $1 \%$ de probabilidade $(\mathrm{p}<.01)$.

Tabela 6 - Número de folhas das mudas de A. polyphylla após a repicagem até a medição final por lâmina de irrigação.

Número de folhas

\begin{tabular}{ccccccccccc}
\hline \multirow{2}{*}{ Lâminas } & $\begin{array}{c}30 \\
\text { dias }\end{array}$ & $\begin{array}{c}60 \\
\text { dias }\end{array}$ & $\begin{array}{c}90 \\
\text { dias }\end{array}$ & $\begin{array}{c}120 \\
\text { dias }\end{array}$ & $\begin{array}{c}150 \\
\text { dias }\end{array}$ & $\begin{array}{c}180 \\
\text { Dias }\end{array}$ & $\begin{array}{c}210 \\
\text { dias }\end{array}$ & $\begin{array}{c}240 \\
\text { dias }\end{array}$ & $\begin{array}{c}270 \\
\text { dias }\end{array}$ & $\begin{array}{c}300 \\
\text { dias }\end{array}$ \\
\hline L1 & 6,37 & 8,86 & 10,66 & 10,72 & 10,09 & 10,79 & 11,33 & 11,66 & 11,39 & 11,49 \\
L2 & 6,65 & 9,02 & 10,79 & 10,86 & 9,79 & 10,33 & 10,90 & 11,22 & 11,41 & 11,40 \\
L3 & 6,63 & 9,07 & 10,86 & 11,20 & 10,21 & 11,01 & 11,74 & 12,04 & 11,95 & 11,65 \\
\hline F \% & 0,80 & 0,35 & 0,23 & 1,02 & 0,93 & 0,68 & 0,69 & 0,86 & 0,55 & 0,08 \\
DMS 5\% & 0,74 & 0,83 & 0,90 & 1,08 & 0,99 & 1,62 & 2,19 & 1,92 & 1,87 & 1,98 \\
CV \% & 11,72 & 9,56 & 8,60 & 10,22 & 10,23 & 15,58 & 19,92 & 16,99 & 16,6 & 17,77 \\
\hline
\end{tabular}

L1= irrigação de 6 minutos, L2= irrigação de 9 minutos e L3= irrigação de 12 minutos.

DMS diferença mínima significativa e CV coeficiente de variação e não significativo.

Morgado et al. (2020), avaliaram o uso de substratos combinados com LEC em diferentes lâminas de água para mudas de embaúba (Cecropia pachystachya). Os resultados mostraram que a formulação de LEC e SC ajudaram no desenvolvimento do número de folhas em S3 (75\% SC e 25\% LEC) e S4 (75\% LEC e $25 \%$ SC) ao longo da lâmina de água L3 - $27 \mathrm{~mm}$. No entanto, ao comparar com os dados do presente estudo, nota-se que S1 (100\% LEC) juntamente com a L3 $(12 \mathrm{~mm})$ foram os que apresentaram melhor desempenho em número de folhas.

Os resultados atingidos para a matéria seca da parte aérea (MSPA) tem o S1 (100\% LEC) como aquele que progrediu significativamente a partir da terceira medição (90 dias), e estes dados se mantiveram até a última medição. Posteriormente, o S2, também apresentou resultados semelhantes, apenas ocorrendo pequenas quedas aos 120, 210 e 300 dias. Já S3 e S4 obtiveram resultados similares entre si ao longo dos meses do experimento. Apenas S5 (100\% SC), a partir dos 60 até 300 dias de medição, diferiu-se dos outros tratamentos com valores inferiores: $0,19 \mathrm{~g}, 0,18 \mathrm{~g}, 0,17 \mathrm{~g}, 0,15 \mathrm{~g}$ e 0,13 $\mathrm{g}$ (Tabela 7). 
Tabela 7 - Avaliação da massa da parte área de mudas de A. polyphylla.

\begin{tabular}{ccccccccccc}
\hline \multicolumn{1}{c}{$\begin{array}{c}\text { Substra- } \\
\text { tos }\end{array}$} & $\begin{array}{c}30 \\
\text { dias }\end{array}$ & $\begin{array}{c}60 \\
\text { dias }\end{array}$ & $\begin{array}{c}90 \\
\text { dias }\end{array}$ & $\begin{array}{c}120 \\
\text { dias }\end{array}$ & $\begin{array}{c}150 \\
\text { dias }\end{array}$ & $\begin{array}{c}180 \\
\text { dias }\end{array}$ & $\begin{array}{c}210 \\
\text { dias }\end{array}$ & $\begin{array}{c}240 \\
\text { Dias }\end{array}$ & $\begin{array}{c}270 \\
\text { dias }\end{array}$ & $\begin{array}{c}300 \\
\text { dias }\end{array}$ \\
\hline $\mathrm{S} 1$ & $0,24 \mathrm{ab}$ & $0,26 \mathrm{bc}$ & $0,31 \mathrm{a}$ & $0,32 \mathrm{a}$ & $0,29 \mathrm{a}$ & $0,31 \mathrm{a}$ & $0,36 \mathrm{a}$ & $0,31 \mathrm{a}$ & $0,35 \mathrm{a}$ & $0,35 \mathrm{a}$ \\
$\mathrm{S} 2$ & $0,32 \mathrm{a}$ & $0,35 \mathrm{a}$ & $0,34 \mathrm{a}$ & $0,28 \mathrm{ab}$ & $0,30 \mathrm{a}$ & $0,31 \mathrm{a}$ & $0,33 \mathrm{ab}$ & $0,32 \mathrm{a}$ & $0,36 \mathrm{a}$ & $0,34 \mathrm{ab}$ \\
$\mathrm{S} 3$ & $0,28 \mathrm{a}$ & $0,31 \mathrm{ab}$ & $0,27 \mathrm{ab}$ & $0,30 \mathrm{a}$ & $0,28 \mathrm{a}$ & $0,27 \mathrm{ab}$ & $0,25 \mathrm{bc}$ & $0,24 \mathrm{ab}$ & $0,27 \mathrm{ab}$ & $0,32 \mathrm{ab}$ \\
$\mathrm{S} 4$ & $0,26 \mathrm{ab}$ & $0,27 \mathrm{~b}$ & $0,23 \mathrm{ab}$ & $0,26 \mathrm{ab}$ & $0,29 \mathrm{a}$ & $0,23 \mathrm{ab}$ & $0,24 \mathrm{c}$ & $0,27 \mathrm{ab}$ & $0,25 \mathrm{~b}$ & $0,24 \mathrm{~b}$ \\
$\mathrm{~S} 5$ & $0,18 \mathrm{~b}$ & $0,19 \mathrm{c}$ & $0,18 \mathrm{~b}$ & $0,17 \mathrm{~b}$ & $0,17 \mathrm{~b}$ & $0,17 \mathrm{~b}$ & $0,17 \mathrm{c}$ & $0,17 \mathrm{~b}$ & $0,15 \mathrm{c}$ & $0,13 \mathrm{c}$ \\
\hline $\mathrm{F} \%$ & $5,34 * *$ & $11,04 * *$ & $5,17 * *$ & $3,39 *$ & $7,17 * *$ & $5,40^{* *}$ & $13,16^{* *}$ & $3,94 * *$ & $14,03 * *$ & $12,92^{* *}$ \\
$\mathrm{DMS}$ & 0.09 & 0.07 & 0.11 & 0.12 & 0.08 & 0.10 & 0.08 & 0.12 & 0.0943 & 0.10 \\
$5 \%$ & & & & & & & & & & \\
$\mathrm{CV} \%$ & 29.5 & 23.35 & 34.72 & 39.03 & 26.23 & 33.58 & 26.24 & 40.64 & 28.94 & 31.85 \\
\hline
\end{tabular}

S1=100\% LEC, S2= $75 \%$ LEC + 25\% SC, S3= 50\% LEC + 50\% SC, S4= 25\% LEC + 75\% SC e S5= 100\% SC. DMS diferença mínima significativa, CV coeficiente de variação e não significativo. * significativo ao nível de $5 \%$ de probabilidade $(.01=<\mathrm{p}<.05)$; $* *$ significativo ao nível de $1 \%$ de probabilidade $(\mathrm{p}<.01)$.

Os resultados demonstram que quanto maior a quantidade de LEC, maior a MSPA (Tabela 7). Resultados semelhantes foram relatadas por Sousa et al. (2020) com mudas de acácia-branca (Moringa oleifera) em diferentes concentrações de LEC. Os autores encontraram as maiores médias na formulação de $60 \%$ LEC e $40 \%$ areia e afirmaram que, quanto maior for à concentração de LEC no substrato, maiores foram as médias de acúmulo de
MSPA.

Na irrigação, a MSPA não demonstrou grandes variações entre si nas diferentes lâminas, apenas a L1 $(0,23$ e 0,23$)$ e L2 $(0,25$ e 0,26$)$ aos 120 e 210 dias. Verificou-se que não houve diferença estatística para nenhuma das lâminas deágua durante o período de amostragem (Tabela 8).

Tabela 8 - Massa da parte aérea das mudas de A. polyphylla por diferentes lâminas de irrigação.

\begin{tabular}{ccccccccccc}
\hline \multicolumn{10}{c}{ Massa da parte aérea das mudas $(\mathrm{g})$} \\
\hline \multirow{2}{*}{ Lâminas } & $\begin{array}{c}30 \\
\text { dias }\end{array}$ & $\begin{array}{c}60 \\
\text { dias }\end{array}$ & $\begin{array}{c}90 \\
\text { dias }\end{array}$ & $\begin{array}{c}120 \\
\text { dias }\end{array}$ & $\begin{array}{c}150 \\
\text { dias }\end{array}$ & $\begin{array}{c}180 \\
\text { dias }\end{array}$ & $\begin{array}{c}210 \\
\text { dias }\end{array}$ & $\begin{array}{c}240 \\
\text { dias }\end{array}$ & $\begin{array}{c}270 \\
\text { dias }\end{array}$ & $\begin{array}{c}300 \\
\text { dias }\end{array}$ \\
\hline L1 & $0,21 \mathrm{a}$ & $0,24 \mathrm{a}$ & $0,22 \mathrm{a}$ & $0,23 \mathrm{~b}$ & $0,24 \mathrm{a}$ & $0,20 \mathrm{a}$ & $0,23 \mathrm{~b}$ & $0,23 \mathrm{a}$ & $0,23 \mathrm{a}$ & $0,23 \mathrm{a}$ \\
L2 & $0,28 \mathrm{a}$ & $0,30 \mathrm{a}$ & $0,26 \mathrm{a}$ & $0,25 \mathrm{ab}$ & $0,28 \mathrm{a}$ & $0,29 \mathrm{a}$ & $0,26 \mathrm{ab}$ & $0,28 \mathrm{a}$ & $0,31 \mathrm{a}$ & $0,29 \mathrm{a}$ \\
L3 & $0,29 \mathrm{a}$ & $0,24 \mathrm{a}$ & $0,32 \mathrm{a}$ & $0,32 \mathrm{a}$ & $0,29 \mathrm{a}$ & $0,30 \mathrm{a}$ & $0,32 \mathrm{a}$ & $0,28 \mathrm{a}$ & $0,29 \mathrm{a}$ & $0,31 \mathrm{a}$ \\
\hline F \% & $4,13 \mathrm{~ns}$ & $1,41 \mathrm{~ns}$ & $3,99 \mathrm{~ns}$ & $6,87^{*}$ & $1,44 \mathrm{~ns}$ & $2,05 \mathrm{~ns}$ & $5,08 \mathrm{~ns}$ & $0,33 \mathrm{~ns}$ & $2,54 \mathrm{~ns}$ & $2,55 \mathrm{~ns}$ \\
DMS 5\% & 0.10 & 0.11 & 0.12 & 0.07 & 0.08 & 0.17 & 0.09 & 0.20 & 0.11 & 0.12 \\
CV \% & 37.80 & 42.02 & 46.57 & 26.9 & 32.33 & 67.12 & 33.98 & 79.55 & 41.08 & 44.18 \\
\hline
\end{tabular}

L1= irrigação de 6 minutos, L2= irrigação de 9 minutos e L3= irrigação de 12 minutos.

DMS diferença mínima significativa e CV coeficiente de variação e não significativo.

* significativo ao nível de $5 \%$ de probabilidade $(.01=<p<.05)$.

Na grande maioria dos estudos, as produções de massa de matéria seca das mudas estão associadas à sua qualidade relativa (Gomes et al., 2003; Lobo et al., 2018; Melo et al., 2018), ou seja, a massa da MSPA passa a ser considerada como bom indicativo de capacidade de resistência a adversidades ambientais pelas plantas já que existe relação direta com a altura e o diâmetro do colo uma vez que os dois representam juntos mais de $80 \%$ da contribuição relativa (Gomes et al., 2002). Já a matéria seca da parte radicular (MSPR) se relaciona à sobrevivência ao plantio em campo (Gomes e Paiva, 2013).

A análise de crescimento, através da quantifica- 
ção da matéria seca apresenta algumas características comportamentais das diversas cultivares existentes. Assim, são os vários processos fisiológicos que afetam o desenvolvimento da planta que estão relacionados com este parâmetro (Brandelero et al., 2002; Benicasa, 2003; Teodoro et al., 2015).

Diferentemente da MSPA que destacou S1
(100\% LEC) como o melhor substrato, a MSPR que manteve resultados superiores ao longo do experimento foi S3 (50\% LEC e 50\% SC). No entanto, S2 novamente demonstrou valores significativamente superior seguido por S1 e S4; já S5 se manteve com valores inferiores apresentando bons resultados apenas aos 60 e 240 dias (Tabela 9).

Tabela 9 - Massa das raízes das mudas de A. polyphylla após desmontagem do experimento e secagem em estufa.

\begin{tabular}{ccccccccccc}
\hline \multicolumn{10}{c}{ Massa da parte aérea das mudas $(\mathrm{g})$} \\
\hline Substratos & $\begin{array}{c}30 \\
\text { dias }\end{array}$ & $\begin{array}{c}60 \\
\text { dias }\end{array}$ & $\begin{array}{c}90 \\
\text { dias }\end{array}$ & $\begin{array}{c}120 \\
\text { Dias }\end{array}$ & $\begin{array}{c}150 \\
\text { Dias }\end{array}$ & $\begin{array}{c}180 \\
\text { dias }\end{array}$ & $\begin{array}{c}210 \\
\text { dias }\end{array}$ & $\begin{array}{c}240 \\
\text { dias }\end{array}$ & $\begin{array}{c}270 \\
\text { dias }\end{array}$ & $\begin{array}{c}300 \\
\text { dias }\end{array}$ \\
\hline S1 & $18,13 \mathrm{ab}$ & $19,39 \mathrm{a}$ & $18,59 \mathrm{ab}$ & $26,96 \mathrm{a}$ & $13,15 \mathrm{Bc}$ & $25,13 \mathrm{ab}$ & $29,17 \mathrm{a}$ & $26,19 \mathrm{a}$ & $23,76 \mathrm{a}$ & $18,56 \mathrm{ab}$ \\
$\mathrm{S} 2$ & $35,14 \mathrm{a}$ & $14,46 \mathrm{a}$ & $31,49 \mathrm{ab}$ & $32,98 \mathrm{a}$ & $31,67 \mathrm{ab}$ & $21,45 \mathrm{ab}$ & $28,13 \mathrm{a}$ & $26,26 \mathrm{a}$ & $20,21 \mathrm{a}$ & $24,41 \mathrm{ab}$ \\
$\mathrm{S} 3$ & $20,18 \mathrm{ab}$ & $27,17 \mathrm{a}$ & $40,16 \mathrm{a}$ & $25,73 \mathrm{a}$ & $40,4 \mathrm{a}$ & $30,97 \mathrm{a}$ & $21,40 \mathrm{ab}$ & $15,71 \mathrm{a}$ & $22,89 \mathrm{a}$ & $25,47 \mathrm{a}$ \\
S4 & $9,50 \mathrm{~b}$ & $14,86 \mathrm{a}$ & $13,43 \mathrm{~b}$ & $18,18 \mathrm{ab}$ & $18,66 \mathrm{bc}$ & $17,91 \mathrm{ab}$ & $15,21 \mathrm{ab}$ & $26,85 \mathrm{a}$ & $17,52 \mathrm{ab}$ & $22,48 \mathrm{ab}$ \\
S5 & $6,60 \mathrm{~b}$ & $7,21 \mathrm{a}$ & $11,10 \mathrm{~b}$ & $4,58 \mathrm{~b}$ & $9,62 \mathrm{c}$ & $9,13 \mathrm{~b}$ & $7,80 \mathrm{~b}$ & $9,04 \mathrm{a}$ & $3,53 \mathrm{~b}$ & $4,26 \mathrm{~b}$ \\
\hline F \% & $4,39 * *$ & $1,97 \mathrm{NS}$ & $3,65 *$ & $5,04 * *$ & $6,07 * *$ & $3,00 *$ & $3,54 *$ & $1,24 \mathrm{NS}$ & $4,31 * *$ & $2,89 *$ \\
DMS 5\% & 21.66 & 21.21 & 26.43 & 19.74 & 21.35 & 19.14 & 19.39 & 29.31 & 16.08 & 20.69 \\
CV \% & 103.19 & 108.94 & 98.23 & 77.68 & 80.26 & 78.07 & 81.33 & 120.16 & 78.03 & 92.72
\end{tabular}

S1 $1=100 \%$ LEC, S2= $75 \%$ LEC + 25\% SC, S3= 50\% LEC + 50\% SC, S4= 25\% LEC + 75\% SC e S5= 100\% SC. DMS diferença mínima significativa, CV coeficiente de variação e ns não significativo. * significativo ao nível de $5 \%$ de probabilidade $(.01=<\mathrm{p}<.05) ; * *$ significativo ao nível de $1 \%$ de probabilidade $(\mathrm{p}<.01)$.

Em função das diferentes lâminas de irrigação, tanto o índice MSPR como o índice MSPA não apresentaram variações nos dados analisados, possibilitando observar que a irrigação não contribuiu de modo considerável nas avaliações desse parâmetro, tal como na altura e número de folhas.

Tabela 10 - Massa da parte da raiz das mudas de A. polyphylla por diferentes lâminas de irrigação.

\begin{tabular}{cccccccccccc}
\hline \multicolumn{10}{c}{ Massa da parte da raiz das mudas $(\mathrm{g})$} \\
\hline \multirow{2}{*}{ Lâminas } & 30 & 60 & 90 & 120 & 150 & 180 & 210 & 240 & 270 & 300 \\
& dias & dias & dias & dias & dias & dias & dias & dias & dias & dias \\
\hline L1 & $13,31 \mathrm{a}$ & $13,35 \mathrm{a}$ & $20,01 \mathrm{a}$ & $22,30 \mathrm{a}$ & $18,36 \mathrm{a}$ & $19,28 \mathrm{a}$ & $14,25 \mathrm{a}$ & $16,27 \mathrm{a}$ & $17,66 \mathrm{a}$ & $13,64 \mathrm{a}$ \\
L2 & $17,85 \mathrm{a}$ & $13,74 \mathrm{a}$ & $22,97 \mathrm{a}$ & $17,78 \mathrm{a}$ & $23,32 \mathrm{a}$ & $14,44 \mathrm{a}$ & $17,07 \mathrm{a}$ & $14,05 \mathrm{a}$ & $14,61 \mathrm{a}$ & $12,93 \mathrm{a}$ \\
L3 & $22,57 \mathrm{a}$ & $22,75 \mathrm{a}$ & $25,89 \mathrm{a}$ & $24,96 \mathrm{a}$ & $26,41 \mathrm{a}$ & $29,03 \mathrm{a}$ & $29,71 \mathrm{a}$ & $32,10 \mathrm{a}$ & $20,46 \mathrm{a}$ & $30,54 \mathrm{a}$ \\
\hline F \% & $1,25 \mathrm{~ns}$ & $0,81 \mathrm{~ns}$ & $0,50 \mathrm{~ns}$ & $0,33 \mathrm{~ns}$ & $0,65 \mathrm{~ns}$ & $3,20 \mathrm{~ns}$ & $3,90 \mathrm{~ns}$ & $2,11 \mathrm{~ns}$ & $0,52 \mathrm{~ns}$ & $4,16 \mathrm{~ns}$ \\
DMS 5\% & 17.94 & 25.59 & 18.00 & 27.40 & 21.77 & 18.03 & 18.09 & 29.40 & 17.66 & 21.20 \\
CV \% & 103.20 & 158.72 & 80.84 & 130.24 & 98.84 & 88.82 & 91.65 & 145.59 & 103.52 & 114.76 \\
\hline
\end{tabular}

L1= irrigação de 6 minutos, L2= irrigação de 9 minutos e L3= irrigação de 12 minutos.

DMS diferença mínima significativa e CV coeficiente de variação e ns não significativo.

Para Rudek et al. (2013), a matéria seca das raízes é um parâmetro muito confiável, além de ser um dos melhores indicadores para determinação de qualidades de mudas. No entanto, tal processo demanda tempo e destruição das mudas, tornando-se inviável na rotina de um viveiro comercial, sendo 
mais adequada em pesquisas científicas. A matéria seca das raízes tem sido largamente estudada e reconhecida por determinar a sobrevivência e o estabelecimento das mudas no campo, pois com maiores áreas radiculares e absorção de nutrientes, consequentemente acarretará o melhor desenvolvimento dos indivíduos no campo. Segundo Padilha et al. (2016), as mudas de diferentes espécies, que apresentam um sistema radicular mais vigoroso em condições ambientais adversas, podem apresentar maior aclimatação do que aqueles com sistema radicular conciso.

Lobo et al. (2021) avaliaram o LEC e o SC através de diferentes misturas na produção de mudas de angico vermelho (Anadenanthera macrocarpa). Estes autores demonstraram que a MSPR foi o único parâmetro de qualidade da muda que apresentou diferença entre os tratamentos, onde S3 $(50 \%$ LEC $+50 \%$ SC) e S5 (100\% SC) obtiveram maior produção que o S1 (100\% LEC). Por outro lado, a partir dos dados aqui apresentados, o presente estudo demonstrou que S3 (50\% LEC + 50\% SC) como o resultado que influenciou positivamente a MSPR das mudas do A. polyphylla, diferente de S5 (100\% SC) que obteve resultados inferiores, exceto nos dias $60(7,21 \mathrm{~g})$ e $240(9,04 \mathrm{~g})$.

Outra questão a se ter em conta, em futuros estudos, é o aproveitamento da água de irrigação em viveiros florestais. Olher et al. (2020) observaram que o uso de água residuária da Estação de Tratamento de Esgoto de Bauru/SP, apresentou efeito positivo significativo no desenvolvimento das mudas de pau viola (Cytharexyllum myrianthum). Segundo estes autores, esse processo, além de melhorar o desenvolvimento das mudas, poderá porventura reduzir os custos de irrigação do viveiro.

\section{CONCLUSÃO}

Os melhores tratamentos para produção de mudas de A. polyphylla em viveiros consistem nas seguintes formulações S1 (100\% LEC), S2 (75\% $\mathrm{LEC}+25 \% \mathrm{SC})$ e S3 (50\% LEC + 50\% SC). Estas combinações resultaram no melhor desenvolvimento das mudas em relação aos tratamentos que continham dosagens superiores de 50\% SC. As diferentes lâminas de irrigação não apresentaram influências significativas em nenhum dos parâmetros avaliados ao longo do experimento.

\section{AGRADECIMENTOS}

Agradecemos ao Viveiro da Associação de Recuperação Florestal e Ecológica (ACIFLORA) pelas facilidades no desenvolvimento da pesquisa e aos dois revisores anônimos pela sugestões e contribuições.

\section{REFERÊNCIAS BIBLIOGRÁFICAS}

Abreu AHM, Leles PSS, Melo LA, Ferreira DHAA, Monteiro FAS. Produção de mudas e crescimento inicial em campo de Enterolobium contortisiliquum produzidas em diferentes recipientes. Revista Floresta, v. 45, n. 1, p. 141-150, 2015. http://dx.doi.org/10.5380/rf

Araújo-Neto JC, Aguiar IB, Ferreira VM, Rodrigues TJD. Armazenamento e requerimento fotoblástico de sementes de Acacia polyphylla DC. Revista Brasileira de Sementes, v. 27, n. 1, p. 115-124, 2005. https://doi.org/10.1590/S010131222005000100014

Araújo-Neto JC, Aguiar IB, Ferreira VM, Paula RC. Caracterização morfológica de frutos e sementes e desenvolvimento pós-seminal de monjoleiro (Acacia polyphylla DC.). Revista Brasileira de Sementes, v. 24, n. 1, p. 203-211, 2002. https://doi.org/10.1590/S0101-31222002000100029

Cabreira GV, Leles PSS, Alonso JM, Abreu AHM, Lopes NF, Santos GR. Biossólido como componente de substrato para produção de mudas florestais. Revista Floresta, v. 47, n. 2, p. 165-176, 2017. https://doi.org/10.5380/rf.v47i1.44291

Camargo R, Maldonado ACD, Dias PAS, Souza MF, França MS. Diagnose foliar em mudas de pinhão-manso (Jatropha Curcas L.) produzidas com biossólido. Revista Brasileira de Engenharia Agrícola e Ambiental, v. 17, n. 3, p. 283-290, 2013. https://doi.org/10.1590/S1415-43662013000300006

Campoe OC, Lanelli C, Stape JL, Cook RL, Mendes JCT, Vivian R. Atlantic forest tree species responses to silvicultural practices in a degraded pasture restoration plantation: From leaf physiology to survival and initial growth. Forest Ecology and Management, v. 313, p. 233-242, 2014. https://doi.org/10.1016/j.foreco.2013.11.016

Chaer GM, Resende AS, Campello EFC, Faria SM, Boddey RM. Nitrogen-fixing legume tree species for the reclamation of severely degraded lands in Brazil. Tree Physiology, v. $31, \quad$ n. $2, \quad$ p. 139-149, 2011. https://doi.org/10.1093/treephys/tpq116

Chu L, He W. Toxic metals in soil due to the land application of sewage sludge in China: Spatiotemporal variations and influencing factors. Science of The Total Environment, 143813 , totenv.2020.143813

Delarmelina WM, Caldeira MVW, Faria JCT, Gonçalves EO. Uso de lodo de esgoto e resíduos orgânicos no crescimento de mudas de Sesbania virgata. Revista Agro@mbiente Online, v. 7, n. 2, p. 184-192, 2013. https://doi.org/10.18227/1982-8470ragro.v7i2.888

Delarmelina WM, Caldeira MVW, Faria JCT, Gonçalves EO, Rocha RLF. Diferentes Substratos para a Produção de Mudas de Sesbania virgata. Floram - Floresta e Ambiente, v. 21, n. 2, p. 224-223, 2014. https://doi.org/10.4322/floram.2014.027.

Gomes JM, Paiva HN. Viveiros florestais: propagação sexuada. Editora UFV, v.1, n.1, p.116, 2013.

Gomes JM, Couto L, Leite HG, Xavier A, Garcia SLR. Crescimento de mudas de Eucalyptus grandis em diferentes tamanhos de tubetes e fertilização N-P-K. Revista Árvore, v. 27, n.2, p. $113-127,2003$.

Gomes JM, Couto L, Leite HG, Xavier A, Garcia SLR. Parâmetros morfológicos na avaliação da qualidade de mudas de 
Eucalyptus grandis. Revista Árvore, Viçosa, v.26, n.6, p. 655-664, 2002

Lobo FL, Oliveira FC, Morgado BT, Siqueira MVBM. Diferentes Misturas de Substratos com Lodo de Esgoto Compostado Enriquecido e Substrato Comercial em Quaresmeira. Journal of Social, Technological and Environmental Science, v.7, n.1, p. 326-340, 2018. https://doi.org/10.21664/2238-8869.2018v7i1.p326-340

Lobo TF, Olher I, Siqueira MVBM, Oliveira FC. Commercial Substrate and sewage sludge composted use in angico vermelho. Fronteiras: Journal of Social, Technological and Environmental Science, v.10, n. 1, p. 325-338, 2021. http://dx.doi.org/10.21664/2238-8869.2021v10i1.p325-338 2021

Melo L, Abreu A, Leles P, Oliveira R, Silva D. Qualidade e crescimento inicial de mudas de Mimosa caesalpiniifolia Benth. produzidas em diferentes volumes de recipientes. Ciência Florestal, v.28, n.1, p. 47-55, 2018. https://doi.org/10.5902/1980509831574

Moreira SF, Santos SDO, Sardinha AS, Junior AP. O lodo de ETE como alternativa para a recuperação do solo em áreas degradadas / ETE sludge as an alternative to soil recovery in degraded areas. Brazilian Applied Science Review, v. 3, n.3, p. 1564-1585, 2019. https://doi.org/10.34115/basrv3n3006

Morgado BT, Olher I, Timo TPC, Rossi MN, Lobo TF, Siqueira MVBM. Growth of Cecropia hololeuca in water blades and substrates formulated whit sewage sludge. Amazonian Journal of Agricultural and Environmental Sciences, v. $63, \quad$ p. 2020. http://dx.doi.org/10.22491/rca.2020.3174

Neves APN, Ferreira DS, Castro DA, Cavallini GS. Avaliação da eficiência de remoção de matéria orgânica em efluente sanitário em função da idade do lodo de reator UASB: um estudo de caso. Journal of Biotechnology and Biodiver-

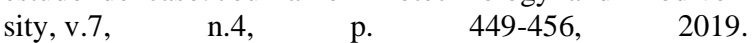
https://doi.org/10.20873/jbb.uft.cemaf.v7n4.neves

Olher I, Siqueira MVBM, Costa GHG, Lobo TF. Crescimento de mudas de pau viola (Cytharexyllum myrianthum) são potencializadas utilizando resíduos do tratamento de esgoto doméstico. Journal of Biotechnology and Biodiversity, v.8, n.4, p. 304-310. 2020. https://doi.org/10.20873/jbb.uft.cemaf.v8n4.olher

Padilha NS, Silva CJ, Pereira SB, Silva JAN, Heid DM, Bottega SP, Scalon SPQ. Crescimento inicial do pinhão-manso submetido a diferentes regimes hídricos em latossolo vermelho distrófico. Ciência Florestal, v. 26, n. 2, p. 513-521, 2016. https://doi.org/10.5902/1980509822752

Rudek A, Garcia AO, Peres FSB. Avaliação da qualidade de mudas de eucalipto pela mensuração da área foliar com o uso de imagens digitais. Enciclopédia Biosfera, Centro Científico Conhecer, v.9, n.17, p. 3775, 2013.

Santana LO, Araújo FM, Souza FLP, Leme MC, Timo TPC, Lobo TF, Siqueira MVBM. Desenvolvimento de mudas de dedaleiro (Lafoensia pacari A.St.-Hil.) mediante diferentes substratos e lâminas de irrigação. Revista Instituto Florestal, v. 31, n.2, p.147-156, 2019.
Scheer MB, Carneiro C, Santos KG. Substratos à base de lodo de esgoto compostado na produção de mudas de Parapiptadenia rigida (Benth.) Brenan. Scientia Forestalis, v. 38, n. 88 , p. 637-644, 2010.

Scheer MB, Carneiro C, Bressan OA, Santos KG. Compostos de lodo de esgoto para a produção de mudas de Anadenanthera colubrina (Vell.) Brenan. Cerne, v. 18, n. 4, p. $613-$ 621, 2012. https://doi.org/10.1590/S010477602012000400011

Sousa AN, Almeida DM, Braga RSS, Barbosa Júnior VC, Santana JAS, Canto JL do. Produção de mudas de Moringa oleífera em diferentes concentrações de lodo de esgoto. Diversitas Journal, v.5, n.3, p. 1504-1522. 2020. https://doi.org/10.17648/diversitas-journal-v5i3-958.

Teodoro PE, Ribeiro LP, Oliveira EP de, Corrêa CCG, Torres FE. Acúmulo de Massa Seca na Soja em Resposta a Aplicação Foliar com Silício sob Condições de Déficit Hídrico. Bioscience Journal, v. 31, n. 1, p. 161-170, 2015. https://doi.org/10.14393/BJ-v31n1a2015-22283

Testezlaf R. Irrigação: métodos, sistemas e aplicações. Campinas: FEAGRI- Faculdade de Engenharia Agrícola, UNICAMP, 203p. 2011.

Trazzi PA, Caldeira MVW, Colombi R, Gonçalves EO. Qualidade de mudas de Murraya paniculata produzidas em diferentes substratos. Revista Floresta, v. 42, n. 3, p. 621-630, 2012. http://dx.doi.org/10.5380/rf.v42i3.19718

Trigueiro RDM, Guerrini IA. Utilização de lodo de esgoto na produção de mudas de Aroeira-pimenteira. Revista Árvore, v. 38 , n. 4, p. 657-665, 2014. https://doi.org/10.1590/S010067622014000400009

Vieira CR, Weber OLS. Produção de mudas de eucalipto em diferentes composições de substratos. REA - Revista de Estudos Ambientais, v.18, n. 2, p.25-34, 2016. https://doi.org/0.7867/1983-1501.2016v18n2p25-34 